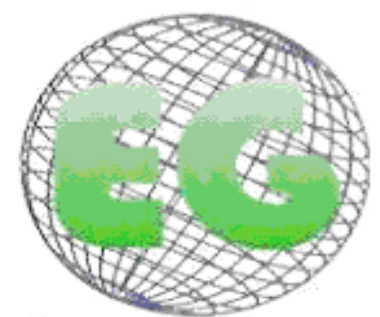

ISSN 1695-6141

$\mathrm{N}^{\circ} 24$

www.um.es/egloball

\title{
CLÍNICA
}

\section{Valoración de los conocimientos del cuidador principal sobre úlceras de presión}

Assessment of the main carer's knowledge or pressure ulcers

\author{
*Martínez López, R., **Ponce Martínez, DE. \\ *Diplomado en Enfermería. Residencia Vista Alegre. Zaragoza. **Diplomada en Enfermería. Hospital \\ Universitario Virgen de la Arrixaca. Murcia. \\ Palabras clave: Cuidador principal, úlceras por presión, conocimientos.
}

Keywords: Main carer; ulcers, UPP (pressure ulcer), knowledge.

\section{RESUMEN}

Introducción. El $30.8 \%$ de la población está representada por personas mayores. Los cambios sociodemográficos están produciendo un incremento progresivo de la población en situación de dependencia, por ello, nace la figura del cuidador.

Objetivos. Evaluar los conocimientos del cuidador principal (CP) en relación a las úlceras por presión (UPP). Estimar los conocimientos sobre los factores de riesgo de las UPP.

Evaluar los métodos de prevención que se utilizan para evitar UPP.

Examinar la identificación de las UPP por parte del CP.

Hipótesis. Existen unos conocimientos deficientes del cuidador principal en relación a las UPP.

Método. Estudio descriptivo, transversal, observacional y prospectivo. Los individuos de estudio han sido 44 cuidadores principales de ancianos dispuestos a colaborar, que hablan y entienden el castellano y cuyo anciano está incluido en el programa de inmovilizados del Centro de Salud de Lorquí.

Para valorar el nivel de conocimientos del CP se ha empleado un cuestionario de elaboración propia.

Resultados La media de edad de los CP es de 53.29 años, la mayoría son mujeres (86\%), con bajo nivel de estudios (57\%). El 92\% realiza cambios posturales.

Discusión y Conclusiones. Los conocimientos del CP sobre: los factores de riesgo de aparición de UPP muestran que la mayoría desconocen factores importantes. En cuanto a los métodos de prevención, el CP conoce algunos pero estos no son suficientes. Y por último, los CP son capaces de reconocer una UPP por el color, dolor, olor y estado de la piel. 


\section{ABSTRACT}

Introduction. Seniors account for $30.8 \%$ of the population. Demographic changes are causing a gradual increase in the population of dependents, and hence the appearance of the figure of the carer.

Objective. To evaluate the main carer's (MC) knowledge of pressure ulcers (UPP).

To estimate the knowledge of risk factors for pressure ulcers.

To evaluate the prevention methods that are being used to prevent pressure ulcers.

To examine the identification of the UPP by the MC.

Hypothesis. There is a deficient knowledge of the main carer in relation to UPP.

Method. Descriptive, observational, and prospective. The study is about 44 main carers of elderly people. All of them should collaborate, to understand and to speak Castilian and their patient must be included in the "Immobilized Program" of the Lorquí Health Center.

We have used a questionnaire specifically designed by us to assess the level of knowledge of the MC.

Results. Mean age of the MC is 53.29 years; the majority are women (86\%), with low educational attainment (57\%). $92 \%$ do postural changes.

Discussion and Conclusions. MC knowledge about the risk factors of pressure ulcers show that most are unaware of important factors. As for prevention methods, the MC knows some, but these are not sufficient. Finally, the MC are able to recognize a UPP from the color, pain, smell and skin condition.

\section{INTRODUCCIÓN}

El envejecimiento de la población es un hecho en la sociedad española que representa el $18.38 \%$ según las estimaciones del 1 de octubre de $2009^{(1)}$. Según las previsiones del censo de 2001 se prevé que en el año 2050 el colectivo de personas mayores represente un $30.8 \%$ de la población ${ }^{(2)}$. La población actual evoluciona hacia un envejecimiento progresivo, con un aumento del número de pacientes ancianos dependientes que permanecen mayoritariamente en sus domicilios y reciben cuidados de su familia a través de la figura del cuidador principal que asume el hecho de cuidar con todas sus consecuencias $^{(3)}$.

Cuando el peso de la atención al anciano dependiente es llevado a cabo por un miembro específico de la familia, a este se le llama comúnmente cuidador principal. Se define como la persona, familiar o allegado con mejores condiciones para asumir responsablemente el cuidado del paciente que asume generalmente de forma voluntaria. En la mayoría de los casos es alguien del entorno familiar, cónyuge o hijos ${ }^{(4)}$. Algunos de los factores por los que surge la figura del cuidador principal son entre otros: el aumento de la esperanza de vida, el envejecimiento de la población, las mejoras del tratamiento, enfermedades crónicas, la reducción de la estancia hospitalaria, la aparición de nuevas afecciones crónicas degenerativas y la incorporación de la mujer al trabajo ${ }^{(5)}$.

La población anciana es una de las áreas donde Dorothea Orem aplica su teoría del déficit de autocuidado, ésta se encuentra dividida en tres subteorías: autocuidado, déficit de autocuidado y sistemas de enfermería ${ }^{(6)}$. La teórica muestra gran interés en esta población ya que es la más vulnerable a padecer enfermedades crónicas y ofrece muchos temas de investigación, incluida la promoción para la salud, el autocuidado para la independencia de los ancianos y el estrés del familiar que suministra el cuidado ${ }^{(7)}$

El estudio de investigación sobre la Evolución de la prevalencia de úlceras por presión en el Hospital de Navarra, cuyos resultados nos aportan información sobre las características de 
los pacientes ulcerados, donde el sexo no es significativo mientras la edad es más elevada; el tiempo de estancia en un hospital también añade un riesgo de aparición ${ }^{(8)}$.

La úlcera por presión (upp) es una lesión de la piel, producida secundariamente a un proceso de isquémico producido por la presión, fricción, cizallamiento o una combinación de las mismas, que puede afectar y necrosar aquellas zonas de la epidermis, dermis, tejido subcutáneo y músculo donde se asientan, pudiendo incluso llegar a afectar a articulaciones y huesos ${ }^{(9)}$. A continuación, procedemos a definir los conceptos anteriormente citados:

Presión: es una fuerza que actúa perpendicular a la piel como consecuencia de la gravedad provocando un aplastamiento tisular entre dos planos, uno perteneciente al paciente y otro externo a él.

Fricción: es una fuerza tangencial que actúa paralelamente a la piel, produciendo roces por movimientos o arrastres.

Cizallamiento: combina los efectos de la presión y fricción, son fuerzas paralelas que se producen cuando dos superficies adyacentes deslizan una sobre otra.

Entre las escalas más utilizadas que miden el riesgo de padecer upp, se encuentran:

- Escala de Norton modificada por el INSALUD (Anexo 1) la más utilizada, es una escala de valoración del riesgo de upp. Cada apartado se puntúa sobre una escala de 1 a 4 , donde 1 significa el estado menos favorable y el 4 el más favorable, con puntuaciones totales que varían de un máximo de 20 a un mínimo de $5^{(10)}$.

- La escala EMINA (Anexo 2), es una escala elaborada y validada por el grupo de enfermería del Instituto Catalán de la Salud para el seguimiento de las UPP (3). Contempla cinco factores de riesgo: estado mental, movilidad, incontinencia, nutrición y actividad, puntuados de 0 a 3 cada uno de ellos. Con la primera letra de cada factor se le ha dado nombre a la escala (EMINA) ${ }^{(10)}$.

- La Escala de Braden (Anexo 3) consta de seis subescalas: percepción sensorial, exposición de la piel a la humedad, actividad física, movilidad, nutrición, roce y peligro de lesiones cutáneas, con una definición exacta de lo que se debe interpretar en cada uno de los apartados de estos subíndices ${ }^{(10)}$.

Según el estudio de investigación Definición y Clasificación de UPP, las localizaciones de mayor incidencia de aparición, de mayor a menor probabilidad de aparición son:

1. Úlceras isquiáticas

2. Úlceras trocantéreas

3. Úlceras sacras

4. Úlceras en talones

5. Úlceras maleolares

6. Úlceras en rodillas

Según el GNEAUPP (Grupo Nacional para el Estudio y Asesoramiento en Úlceras Por Presión y Heridas Crónicas) las upp se clasifican en estadios según las estructuras afectadas de los tejidos: 
- Estadío I: Alteración observable en la piel íntegra relacionada con la presión que se manifiesta por un eritema cutáneo que no palidece al presionar; en pieles oscuras puede presentar tonos rojos, azules o morados. En comparación con un área adyacente u opuesta del cuerpo no sometida a presión puede incluir cambios en unos o más de los siguientes aspectos:

- Temperatura de la piel (caliente o fría).

- Consistencia del tejido (edema o induración).

- Sensaciones (dolor, escozor).

- Estadío II: Pérdida parcial del grosor de la piel que afecta a epidermis, dermis o ambas. Úlcera superficial que tiene aspecto de abrasión, ampolla o cráter superficial.

- Estadío III: Pérdida total del grosor de la piel que implica lesión o necrosis del tejido subcutáneo que puede extenderse hacia abajo pero no por la fascia subyacente.

- Estadío IV: Pérdida total del grosor de la piel con destrucción extensa, necrosis del tejido o lesión en músculo, hueso o estructura de sostén (tendón, capsula articular, etc.). en este estadio como en el III, pueden presentarse lesiones con cavernas, tunelizaciones y trayectos sinuosos ${ }^{(10)}$.

\section{Estos son los cuidados específicos de la prevención:}

\section{- Piel}

- Examine el estado de la piel al menos una vez al día.

- Mantenga la piel del anciano en todo momento limpia y seca.

$\checkmark$ Utilice jabones o sustancias limpiadoras con potencial irritativo bajo.

$\checkmark$ Lave la piel con agua tibia, aclare y realice un secado meticuloso sin fricción.

$\checkmark$ No utilice sobre la piel ningún tipo de alcoholes (de romero, colonias, etc.).

$\checkmark$ Aplique cremas hidratantes, procurando su completa absorción.

- Valore la posibilidad de utilizar productos con ácidos grasos hiperoxigenados en las zonas de riesgo de desarrollo de úlceras por presión, cuya piel esté intacta.

- No realice masajes directamente sobre prominencias óseas.

- Dedique una atención especial a las zonas donde existieron lesiones por presión con anterioridad, al presentar un elevado riesgo de aparición de nuevas lesiones (recurrencia).

\section{- Exceso de humedad: Incontinencia, transpiración o drenajes de heridas}

- Valore y trate los diferentes procesos que puedan originar un exceso de humedad en la piel del paciente: incontinencia, sudoración profusa, drenajes y exudado de heridas.

- Para ello puede utilizar productos barrera que no contengan alcohol y que protegen contra exudados y adhesivos.

\section{- Manejo de la presión}

- Para minimizar el efecto de la presión como causa de úlceras por presión habrán de considerarse cuatro elementos: la movilización, los cambios posturales, la utilización de superficies especiales de apoyo y la protección local ante la presión. 


\section{- Movilización}

- Elabore un plan de cuidados que fomente y mejore la movilidad y actividad del paciente.

\section{- Cambios posturales}

- Realice cambios posturales:

$\checkmark$ Cada 2-3 horas a los pacientes encamados, siguiendo una rotación programada e individualizada.

$\checkmark$ En períodos de sedestación se efectuarán movilizaciones horarias. Si puede realizarlo autónomamente, enseñe al paciente a movilizarse cada quince minutos (cambios de postura y/o posiciones).

- En la realización de los cambios posturales tenga presente los siguientes puntos:

$\checkmark$ Evite en lo posible apoyar directamente al paciente sobre sus lesiones.

$\checkmark$ Siga las recomendaciones de salud laboral sobre manejo de pesos y cargas.

$\checkmark$ Mantenga el alineamiento corporal, la distribución del peso y el equilibrio del paciente.

$\checkmark$ Evite el contacto directo de las prominencias óseas entre si.

$\checkmark$ Evite el arrastre.

$\checkmark$ Realice las movilizaciones reduciendo las fuerzas tangenciales y la fricción.

$\checkmark$ El decúbito lateral, no sobrepase los 30․

$\checkmark$ Si fuera necesario, eleve la cabecera de la cama lo mínimo posible (máximo $30^{\circ}$ ) y durante el mismo tiempo.

$\checkmark$ No utilizar flotadores.

\section{- Superficies especiales de apoyo}

- Nos referiremos a superficies especiales de apoyo, como aquellas superficies que han sido diseñadas específicamente para el manejo de la presión. En este sentido, el fabricante debería aportar información fundamentada acerca de sus indicaciones de uso (tipo de pacientes a los que va destinado, características de las lesiones de los pacientes tributarios de su uso, así como parámetros de efectividad respecto del alivio-reducción de la presión).

- Utilice, preferentemente en todos los niveles asistenciales, una superficie de apoyo adecuada según el riesgo detectado de desarrollar úlceras por presión y la situación clínica del paciente.

$\checkmark$ Paciente de riesgo bajo: Preferentemente superficies estáticas (colchonetas- cojines estáticos de aire, colchonetas-colchones-cojines de fibras especiales, colchones de espuma especiales, colchonetas-cojines viscoelásticos...).

$\checkmark$ Pacientes de riesgo medio: Preferentemente superficies dinámicas (colchonetas alternantes de aire de celdas medias,...) o superficies estáticas de altas prestaciones (colchones de espuma especiales, colchones- colchonetas viscoelásticos...). 
$\checkmark$ Pacientes de riesgo alto: Superficies dinámicas (colchones de aire alternante, colchonetas de aire alternante de grandes celdas...).

$\checkmark$ Los pacientes de riesgo medio y alto deberán utilizar un cojín con capacidad de reducción de la presión mientras estén en sedestación.

$\checkmark$ Existen en el mercado superficies especiales diseñadas para pacientes geriátricos.

$\checkmark$ Considere siempre a las superficies especiales como un material complementario que no sustituye al resto de cuidados (movilización y cambios posturales). ${ }^{(11)}$

Tras la revisión bibliográfica planteamos la siguiente hipótesis: Existen unos conocimientos deficientes del cuidador principal en relación a las úlceras por presión.

El $50 \%$ de las UPP, están localizadas a nivel domiciliario con una incidencia del $4.3 \%$ y una prevalencia del $12.9 \%{ }^{{ }^{(9)}}$. Precisamente por este hecho, y por la evolución del envejecimiento de la población, la atención que proporciona el cuidador principal es insustituible ${ }^{(2)}$, por ello, nos planteamos como objetivo principal: Evaluar los conocimientos del cuidador principal en relación a las upp. Como objetivos secundarios:

- Estimar los conocimientos sobre los factores de riesgo de las úlceras por presión.

- Evaluar los métodos de prevención que se utilizan para evitar úlceras por presión.

- Examinar la identificación de las úlceras por presión por parte del cuidador principal.

\section{MATERIAL Y MÉTODOS}

Se ha realizado un estudio descriptivo, transversal, observacional y prospectivo.

El estudio consta de una primera parte en la que se han recogido datos bibliográficos, tanto de artículos como de monografías que hablasen sobre el objeto de estudio. Finalizada esta fase, se tomó contacto con el Centro de Salud de Lorquí a finales de enero de 2010, donde expusimos nuestra idea y les pareció muy interesante, también nos dieron consejo sobre los grupos de mayor interés para nuestro trabajo, concretamente escogimos el programa de inmovilizados por su alto riesgo de deterioro de la integridad cutánea; éste está compuesto por un total de 60 pacientes (población de estudio).

Lorquí es un municipio de la Región de Murcia, perteneciente a la comarca de la Vega Media del Segura se ha identificado históricamente por ser una villa agrícola. Dista $18 \mathrm{~km}$ de la ciudad de Murcia y su altitud media es de 89 metros. Su extensión es de 15,8 km², tiene una población de 7000 habitantes (abril de 2010) de los cuales 3.380 son mujeres.

El siguiente paso fue, acudir a los domicilios acompañados por alguien del equipo que nos presentasen, con el fin de no intimidar, para la realización de la encuesta (anexo 4) en la ausencia del enfermero del equipo. Primero, realizamos un pilotaje del cuestionario para modificar las partes que los cuidadores no comprendían del todo.

Con respecto a la muestra " $n$ " ha sido de 44 personas, los criterios de inclusión fueron:

- Que fuesen cuidadores principales de ancianos.

- Que estuviesen dispuestos a colaborar. 
- Que hablen y entiendan el castellano.

- Que el anciano estuviese incluido en el Programa de Inmovilizados en el Centro de Salud de Lorquí.

No se ha utilizado técnica de muestreo, al coincidir los sujetos de estudio con la muestra, ya que, se intentará estudiar a toda la población que cumpla los criterios al no ser un número excesivamente elevado.

Variables del estudio:

- Edad.

- Sexo.

- Parentesco del cuidador con el enfermo.

- Nivel de estudios se ofrecían tres opciones, con el fin de limitar las posibilidades.

- Enfermedades de riesgo, se evalúa la presencia o ausencia de enfermedades que guarden relación con el hecho de que aparezcan úlceras.

- Presencia de úlceras por presión de cualquier grado.

- Conocimiento zonas comunes de aparición de upp, este ítem será codificado contrastando las respuestas obtenidas con las respuestas correctas, de esta manera juzgaremos si conoce las zonas de aparición.

- Movilidad del paciente.

- Si la respuesta anterior es negativa, preguntamos el tiempo encamado que se expresará en meses.

- Factor de riesgo, se ofreció una pregunta abierta y se agruparon las respuestas más comunes.

- Métodos de prevención.

- Cambios posturales.

- Tiempo entre cambios posturales, se medirá en horas.

- Uso de superficies de apoyo, si la respuesta es afirmativa se dejará que responda abiertamente para obtener información sobre el o los tipos de superficies de apoyo que utiliza puntuando si son correctas o no.

- Soporte nutricional, si la respuesta es afirmativa daremos la oportunidad de expresarse abiertamente sin dar otras opciones.

- En este último ítem 19, queremos evaluar el conocimiento sobre la identificación de la aparición de una upp, no ofreciendo opciones dejando una respuesta abierta favoreciendo un ambiente de confianza. Cuando tengamos esta respuesta se cotejará con la información de la revisión bibliográfica dando la validez suficiente o insuficiente del conocimiento.

El instrumento de investigación que se ha empleado ha sido una entrevista cerrada, que constaba de 19 ítems en total, 18 preguntas cerradas y 1 abierta, las preguntas han sido de elaboración propia, de manera que la única validación que hemos obtenido ha sido la propia de realizar en un principio una encuesta de pilotaje y posteriormente la modificación de esta obteniendo la entrevista final que ha sido elaborada a partir de la revisión bibliográfica y los objetivos a estudiar. 


\section{RESULTADOS}

Se recogieron 44 cuestionarios válidos.

\section{Edad:}

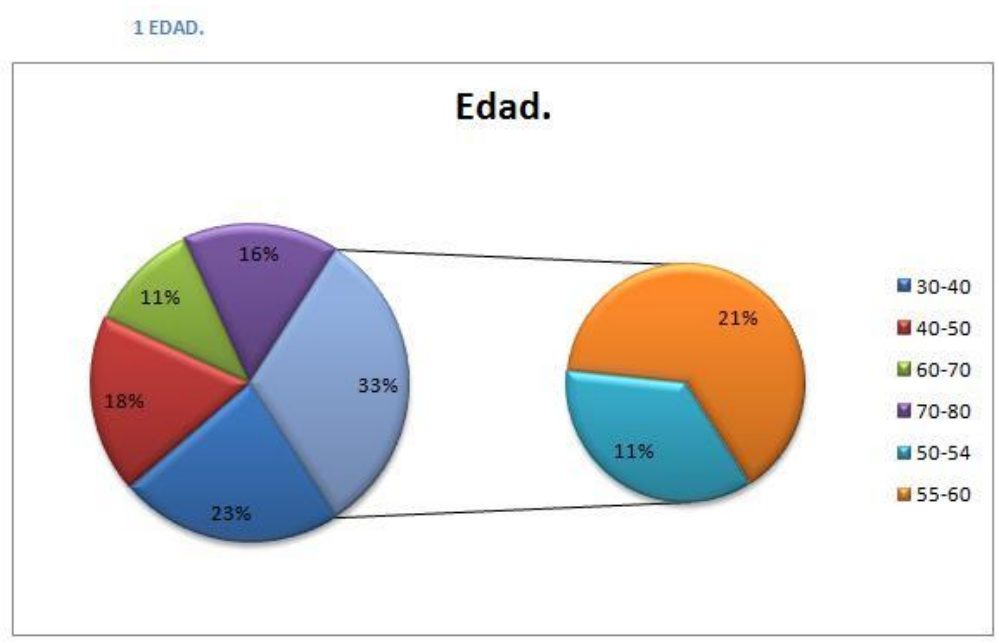

Se decidió englobar las edades en intervalos para su representación gráfica. Se desglosan, las edades comprendidas entre 50 y 60 años por ser estas las mayoritarias (32\%).

La edad, resulta un dato muy interesante, la media de edad en los cuidadores encuestados es de 53.29 años. Esta cifra significa que la responsabilidad del cuidado recae sobre una persona de edad avanzada, que probablemente tenga algún problema de salud y del que en la mayoría de las ocasiones va a depender más de una persona, y no solo en el cuidado sino también económicamente.

\section{Sexo:}

2 SEXO.

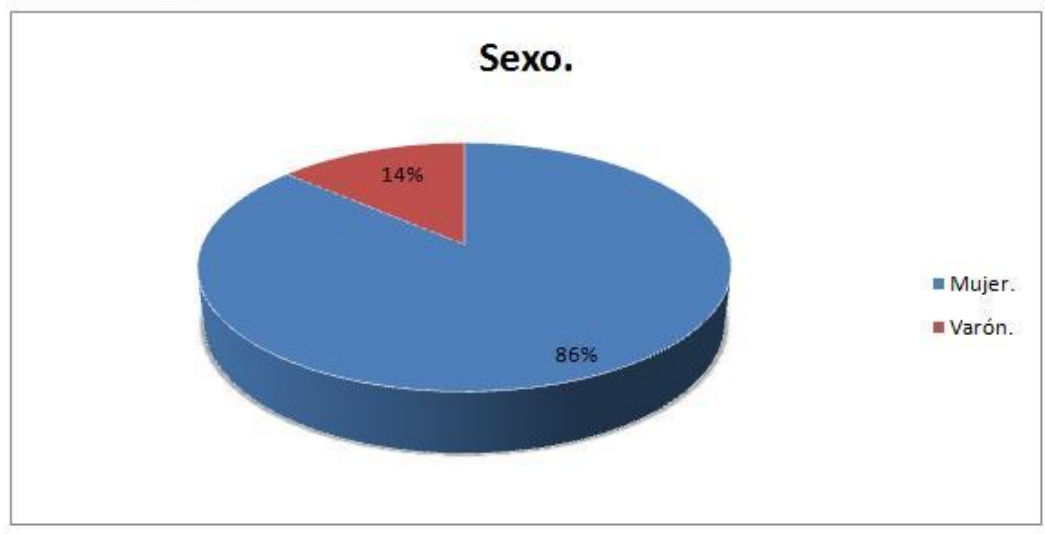

Con respecto a la gráfica, se representa la proporción entre hombres y mujeres que asumen el rol de cuidadores principales, aquí, vemos un dato muy importante. En gran medida son mujeres las que asumen el cuidado, esto debemos tenerlo en cuenta para valorar las necesidades que pueden surgirle, ya que en la actualidad suele se, la mujer la que se hace también responsable de las tareas domésticas. Esto puede ocasionar el agotamiento del 
cuidador principal en el caso de que éste no reciba apoyo de otros miembros del núcleo familiar.

\section{Parentesco del cuidador con el enfermo:}

3 PARENTESCO DEL CUIDADOR CON EL ENFERMO.

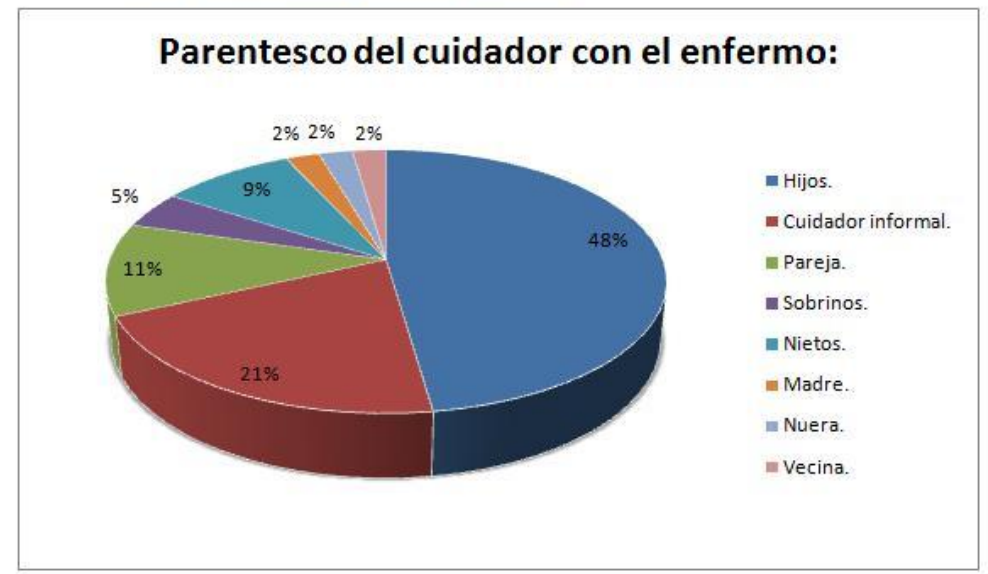

Como se aprecia en el gráfico, el parentesco que une a la significativa cantidad de $48 \%$ de los cuidadores con sus enfermos es el materno-filial, es decir, lo más común en la muestra, es que las hijas asuman el cuidado de los padres cuando estos enferman. También es destacable el porcentaje de cuidadores informales (21\%), esto podría interpretarse como la ocupación laboral excesiva por parte de los hijos, ya que el cuidador informal no guarda ningún parentesco con respecto al enfermo y además su labor está remunerada.

\section{Nivel de estudios:}

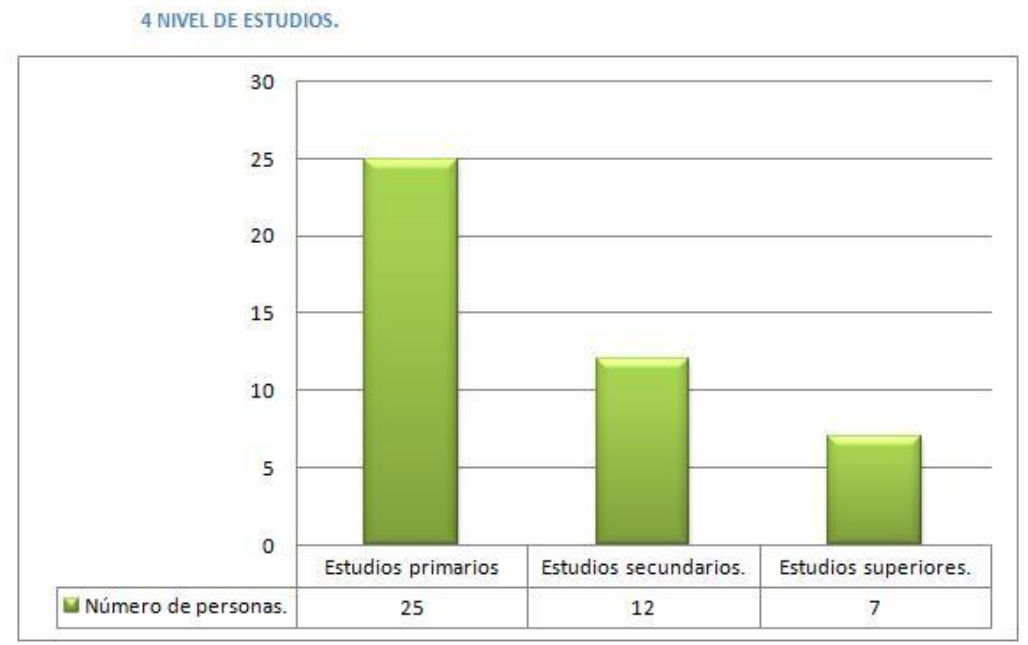

Existe un elevado porcentaje de los cuidadores que presenta un bajo nivel de estudios.

Según la representación gráfica, el nivel de estudios más representativo es el de estudios primarios con un porcentaje de $57 \%$ frente a un $16 \%$ de los cuidadores con estudios superiores. Esto nos pareció interesante para comprobar si había algún tipo de relación con 
el conocimiento o desconocimiento de los factores de riesgo, los métodos de prevención y la identificación de las upp. El elevado porcentaje de cuidadores con estudios primarios se debe a que la media de edad está muy elevada y antiguamente, por esta razón, no se podía optar con tanta facilidad a los estudios como en la actualidad.

\section{Enfermedades relacionadas}

5 ENFERMEDADES RELACIONADAS.

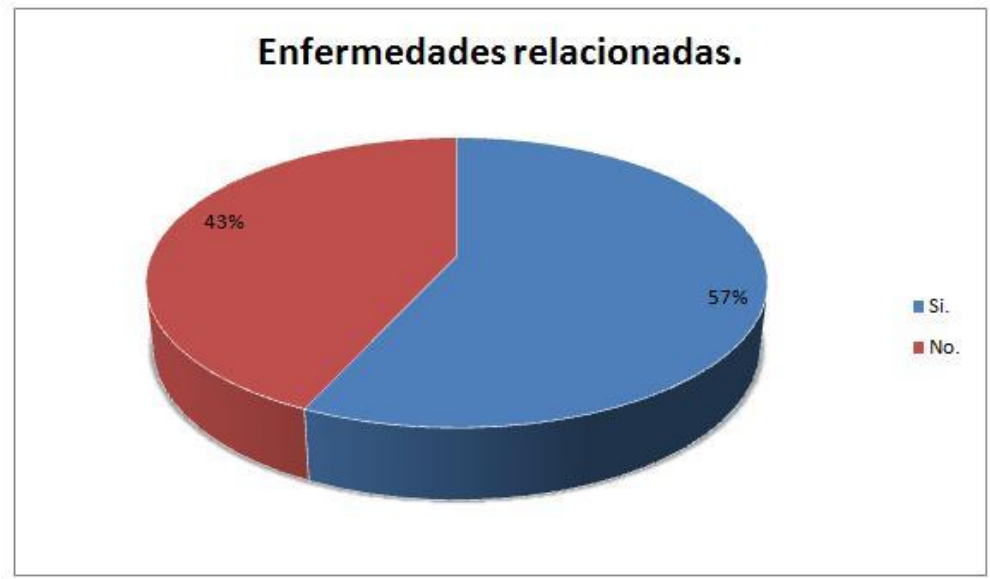

Tal como aparece en la gráfica, sobre enfermedades relacionadas con la aparición de las úlceras por presión, el $57 \%$ de las personas cuidadas sufrían algún tipo de enfermedad como diabetes mellitus $\mathrm{y} / 0$ problemas vasculares 0 venosos que las hacían más susceptibles a padecer upp. Una vez contestada esta pregunta, si la respuesta era afirmativa se les preguntaba si creían que podría haber una relación entre esta enfermedad padecida por el anciano y la aparición de estas heridas, el 90\% contestaron que sí guardaban relación ambos conceptos.

\section{Enfermedades de Riesgo:}

6 FELACION DE ENFERMEDADES DE RIESGO CON APARICIÓN DE UPP.

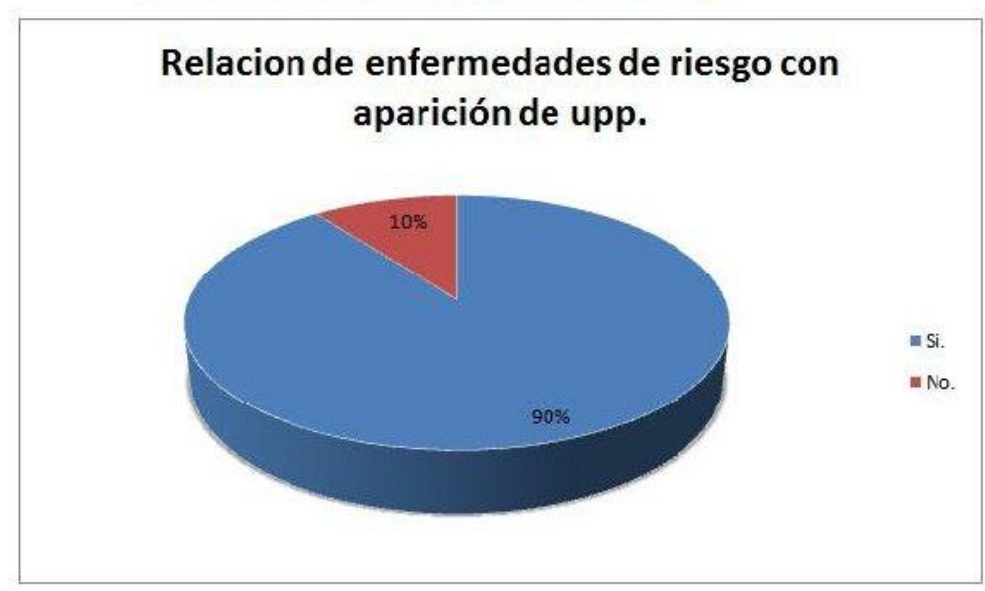




\section{Presencia de upp:}

7 PRESENCIA DE UPP.

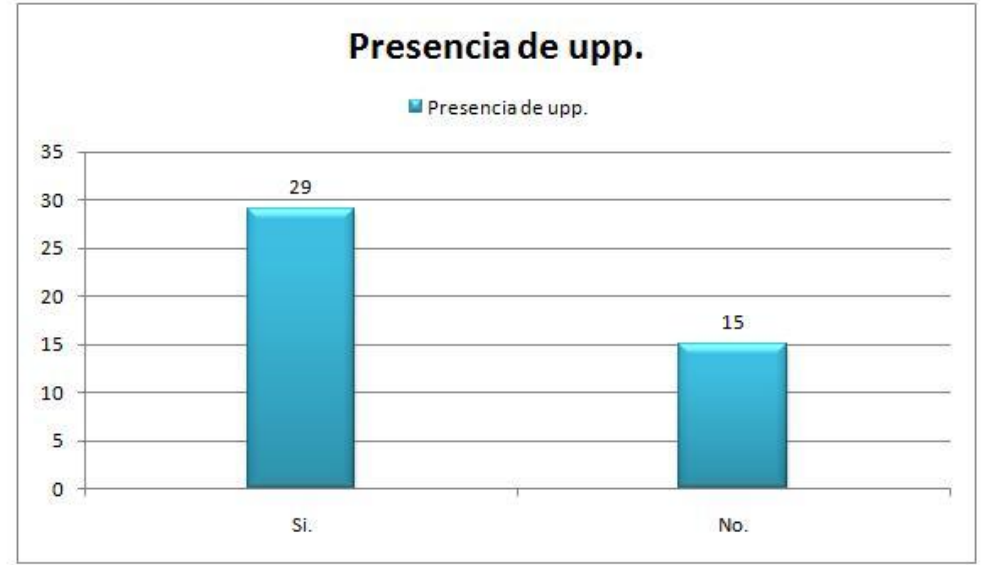

De un total de 44 encuestados, 29 ancianos padecían o habían padecido úlceras por presión.

En este ítem, el $66 \%$ de las personas encuestadas aseguraron que la persona a la que dan los cuidados había sufrido o sufría úlceras por presión durante el tiempo en el que estaban siendo cuidados. Este elevado porcentaje afirma que estas heridas son un gran problema por su frecuente presencia y difícil curación.

\section{Zonas comunes de aparición:}

8 ZONAS COMUNES DE APARICIÓN.

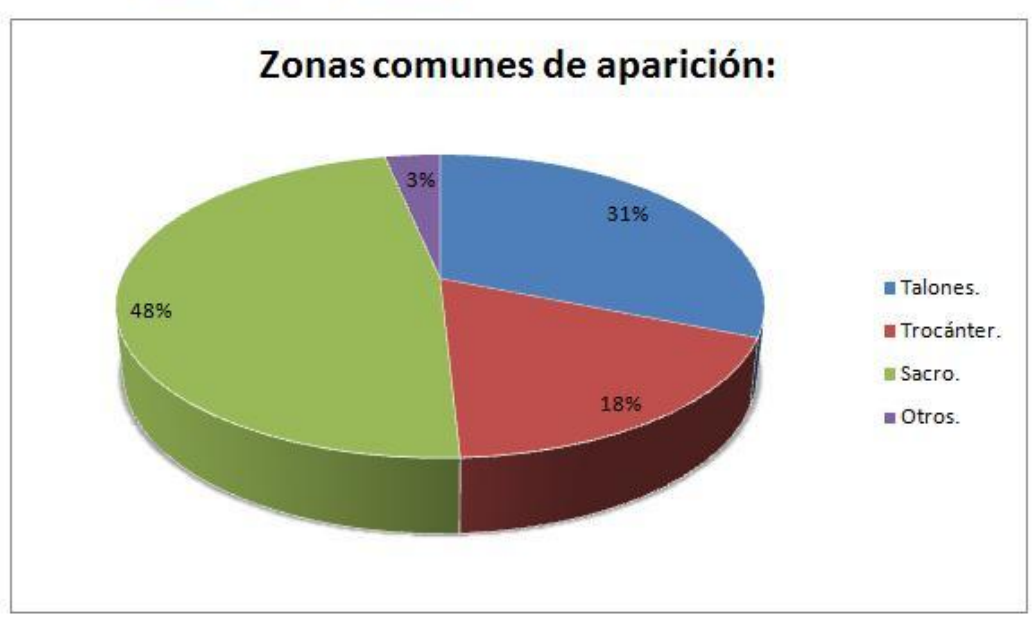

A consecuencia de la pregunta número 6 , se consideró importante interpelar a los cuidadores sobre los conocimientos que tenían acerca de las zonas de común aparición, ya que sobre éstas, los cuidadores son los encargados de poner un especial cuidado para la prevención. Las respuestas de los cuidadores fueron: mayoritariamente el sacro con un $48 \%$, seguido de los talones con un total de $32 \%$, un $18 \%$ contestaron los trocánteres como zona común de aparición y sólo un $2 \%$ del total dieron a conocer otras zonas de riesgo de aparición de upp, como son oreja, hombro y codos. 


\section{Movilidad del paciente:}

9 MOVILIDAD DEL PACIENTE.

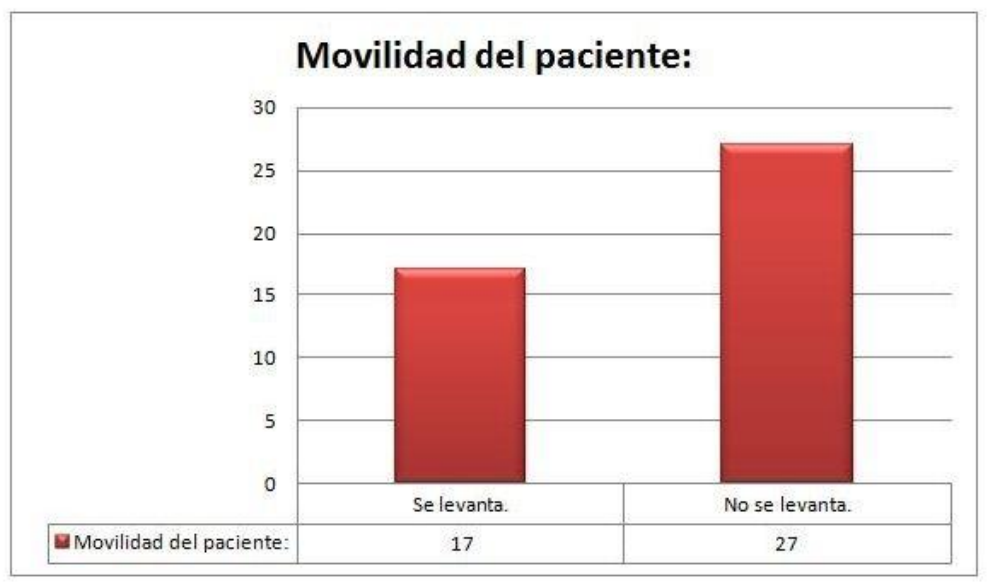

En el gráfico, sobre la movilidad del paciente, la respuesta es mayoritariamente "no", es decir, que 27 de los enfermos se encontraban encamados y los 17 restantes tenían una vida cama sillón.

10.Tiempo de inmovilización:

10 TIEMPO DE INMOVILIZACIÓN.

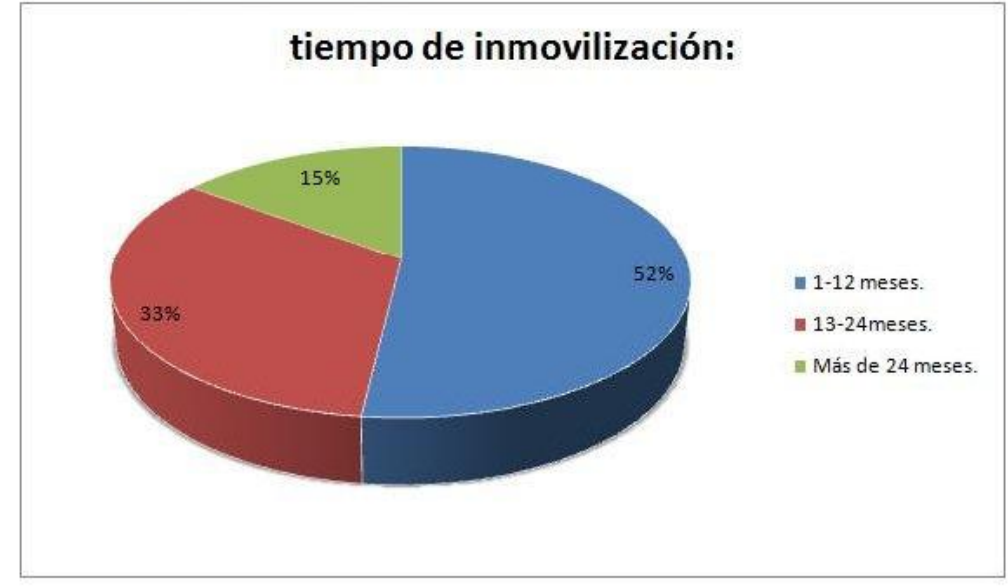

Para su codificación se agruparon por meses, en intervalos de 12 meses. Se observa un elevado porcentaje en los enfermos encamados en el primer intervalo (52\%).

A la luz de la pregunta anterior, consideramos preguntar el tiempo de inmovilización por ser uno de los factores influyentes a la hora de la aparición de las upp. De los 27 inmovilizados, un $52 \%$ se encontraban en situación de encamados en el intervalo de 1 a 12 meses, el $33 \%$ guardaban esta situación de 13 a 24 meses y por último, un mínimo porcentaje, tan solo un $15 \%$ se hallaban de esta manera más de 24 meses. 


\section{Factores de Riesgo:}

11 FACTORES DE RIESGO.

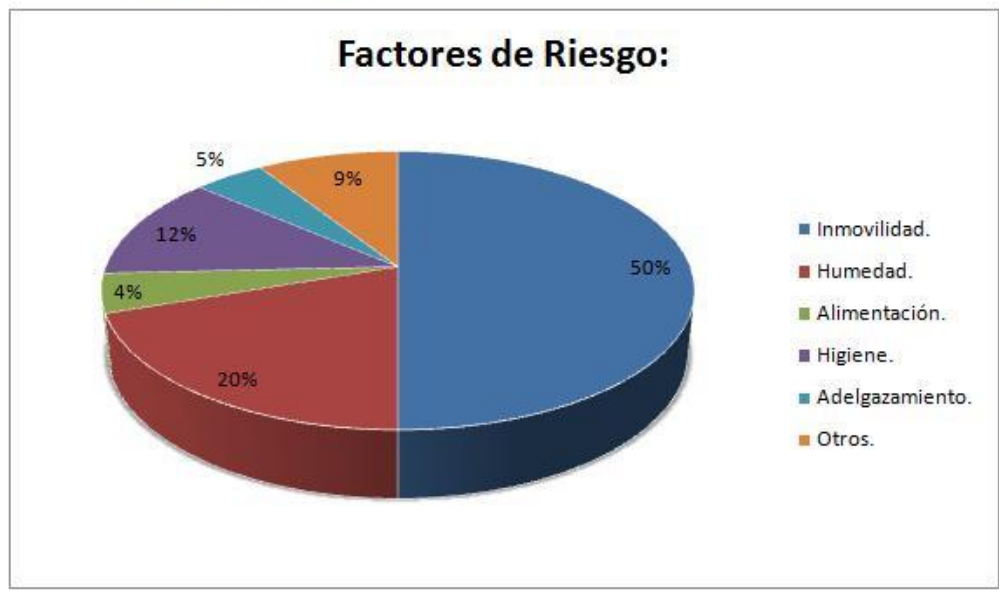

En esta variable, se preguntaba sobre el conocimiento de factores de riesgo, ofreciendo una pregunta abierta. Para su codificación se recabaron las respuestas más comunes.

Este ítem, se consideró importante para poder alcanzar uno de nuestros objetivos, estimar los conocimientos sobre los factores de riesgo de las úlceras por presión. Se muestra la distribución de los diferentes factores que pueden ayudar al brote o empeoramiento de las upp. Las respuestas se dividieron: el $50 \%$ de los cuidadores afirmaron que las upp aparecen como consecuencia de la inmovilidad, un $20 \%$ aseguraron que la principal causa es la humedad que puede estar producida por el uso de pañales, un $12 \%$ piensan que la higiene tiene un papel primordial, un $5 \%$ lo achacan a el adelgazamiento de la piel por el hecho de ser mayores, un $4 \%$ opina que la alimentación es un factor importante. Por último, el $9 \%$ restante concibe otros factores de riesgo como: edad avanzada, enfermedad, traumatismos y fármacos.

\section{Métodos de prevención:}

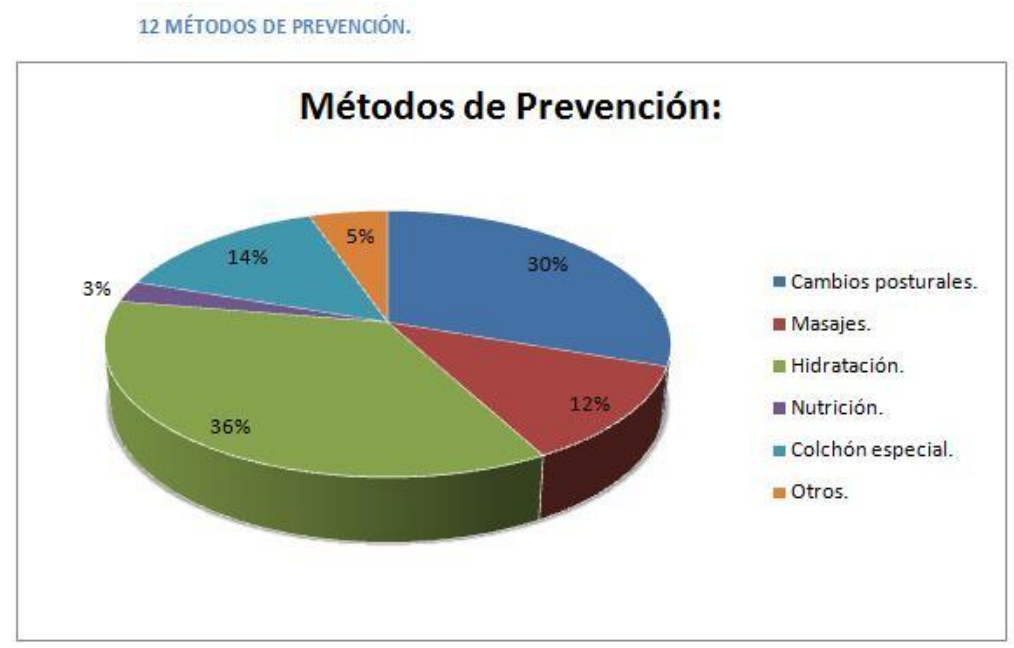

Al igual que la gráfica anterior, se ofreció una pregunta abierta en la que se interpelaba sobre los métodos de prevención utilizados por el cuidador para evitar la aparición de las upp. 
Este gráfico da respuesta a otro de nuestros objetivos, evaluar los métodos de prevención que se utilizan para evitar úlceras por presión. Se reparten los diferentes métodos que ayudan en la prevención. Las respuestas se dividieron en: el $36 \%$ utilizaban como método de prevención principal la hidratación, ya fuera con cremas hidratantes o ácidos grasos hiperoxigenados, el $30 \%$ de los encuestados daban mucha importancia a los cambios posturales realizándolos asiduamente, el 14\% hacían uso de un colchón especial (antiescaras), un $12 \%$ aseguró que masajear las zonas afectadas es una buena forma de prevención porque ayuda a la circulación, sólo un 3\% daba importancia a la nutrición como medida de prevención. Por último, un $5 \%$ utiliza otros métodos como son: higiene, elevación de los miembros inferiores.

\section{Cambios posturales:}

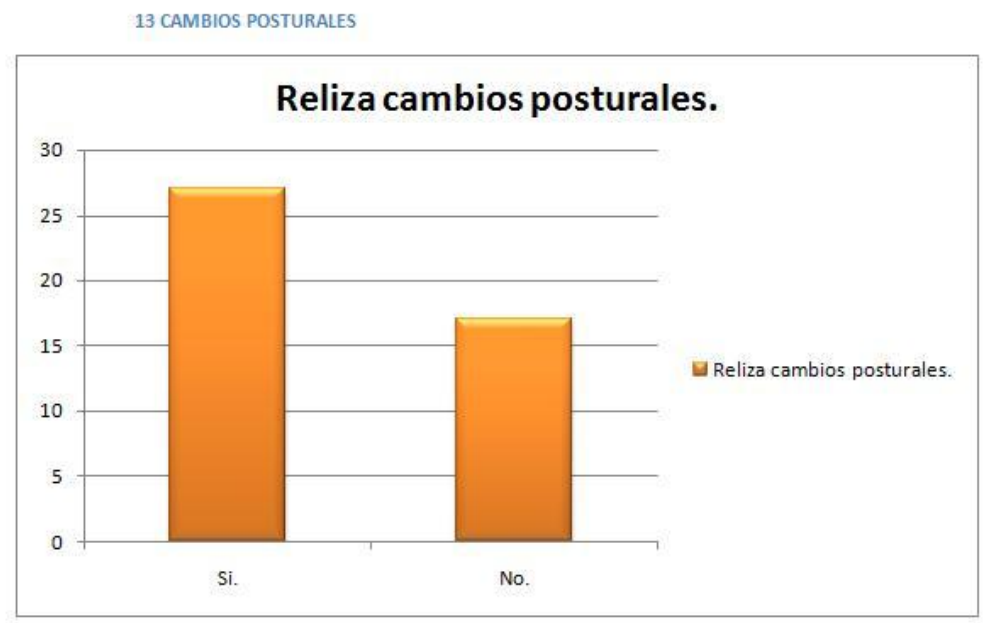

En el gráfico número 12, preguntábamos sobre la realización de cambios posturales, donde la respuesta más frecuente fue la afirmativa con un $61 \%$ frente al $39 \%$ de los cuidadores que no realizaban cambios posturales refiriendo que estos no surtían efecto.

\section{Tiempo entre cambios posturales:}

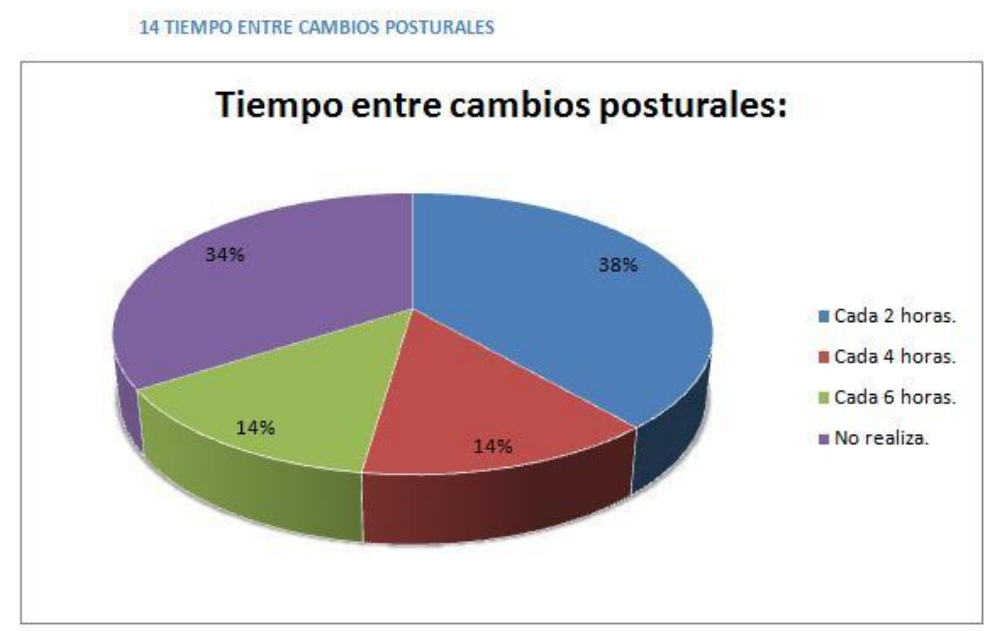

Con todo lo anteriormente relacionado, debemos valorar la frecuencia con la que se realizan los cambios posturales para valorar su efectividad. En el gráfico no 14, vemos claramente los 
porcentajes en intervalos de dos horas. Es significativo que el porcentaje más alto sea de $38 \%$ y se corresponda con el intervalo de cada dos horas, lo cual, bibliográficamente se considera un método de prevención eficiente, los intervalos de cada 4 y cada 6 horas corresponden a un $14 \%$ cada uno. Por último, un $34 \%$ de los encuestados comentaron que no realizaban estos cambios de posición.

\section{Uso de superficies de apoyo:}

15 USO DE SUPERFICIES DE APOYO

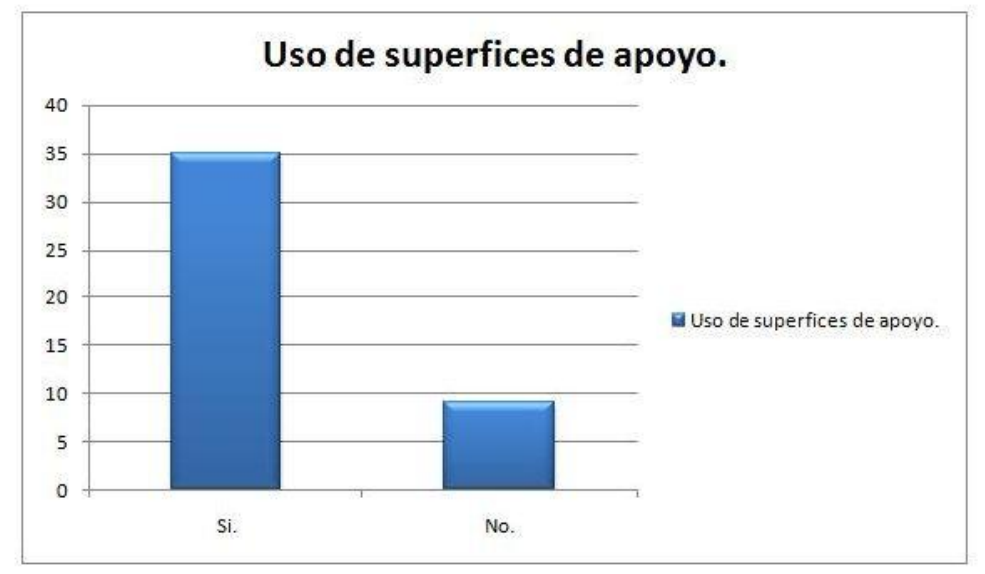

Nos pareció importante este ítem, por la facilidad de proteger las prominencias óseas, únicamente acolchando la zona. El gráfico muestra los resultados sobre el uso de superficies de apoyo, revelando que 35 de los cuidadores poseían algún tipo de accesorio especial para la prevención de las úlceras, frente a 9 personas que no las utilizaban por la creencia de no ser efectivas.

\section{Tipos de superficie de apoyo}

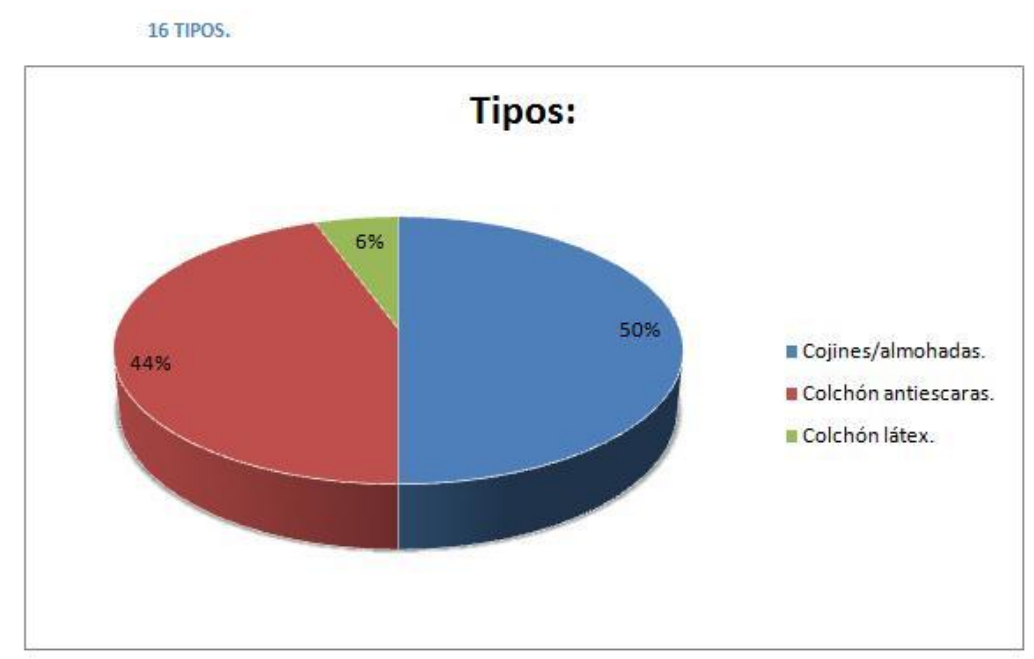

Del total de 35 personas que contestaron afirmativamente al ítem anterior, se les interrogó sobre el tipo de superficie de apoyo que utilizaban para proteger las zonas de riesgo. El $50 \%$ hacía uso de cojines o almohadas, el $44 \%$ poseían un colchón especial antiescaras y solamente un $6 \%$ contaba con un colchón de látex. 


\section{Soporte nutricional:}

17 SOPORTE NUTRICIONAL.

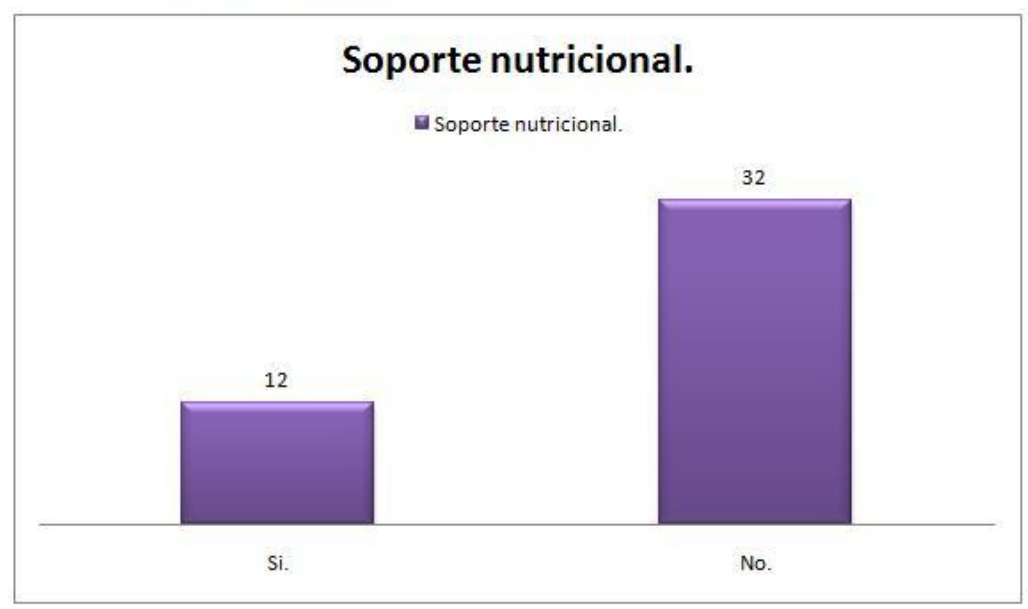

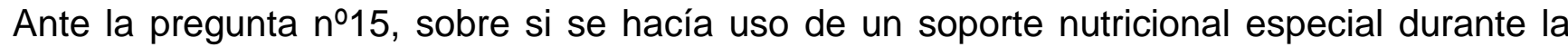
curación de la upp, sólo 12 personas (27\%) respondieron afirmativamente, mientras el resto, 32 personas $(73 \%)$ contestaron de forma negativa porque pensaban que no era importante para la curación.

18. Tipo de alimentción:

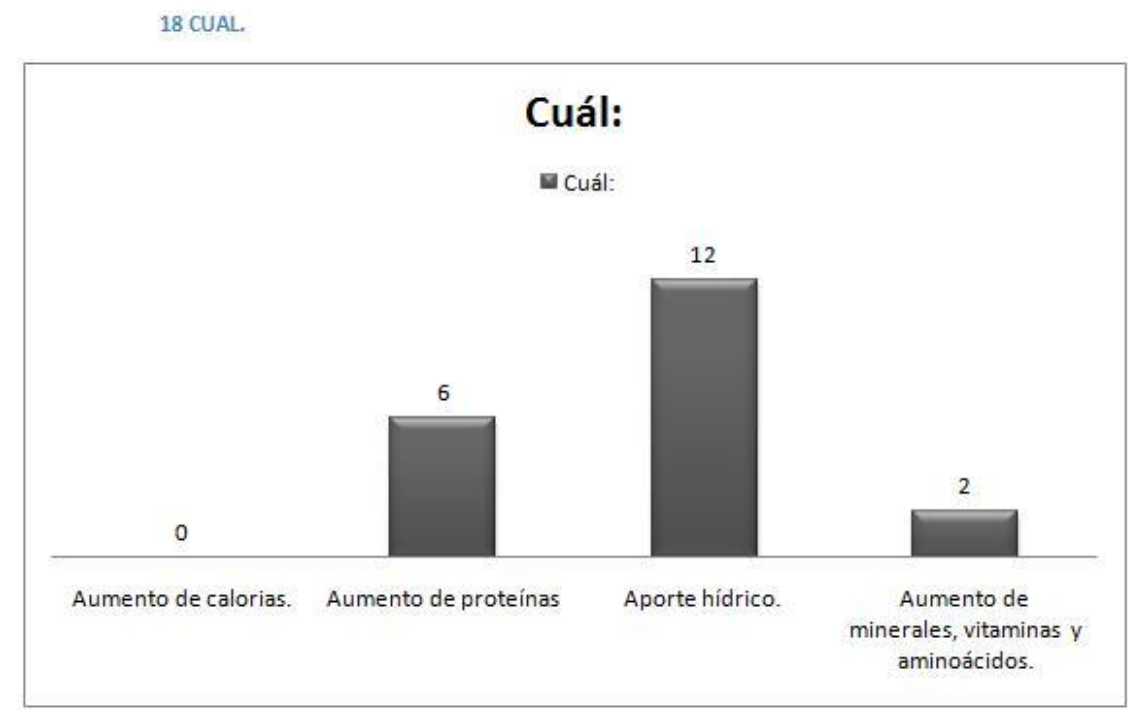

A los 12 cuidadores que utilizaban la alimentación como soporte, se les preguntó sobre el tipo de alimentación que le administraban a su enfermo. Las respuestas fueron: $60 \%$ utilizaban como soporte adicional un aporte hídrico extra, un 30\% consideraba el aumento de proteínas como un soporte nutricional esencial, un $10 \%$ aportaban vitaminas, minerales y aminoácidos. Ninguno de los encuestados consideró efectivo el aporte de calorías.

\section{9. ¿Cómo se encuentra la piel cuando empieza a formarse la upp?}

Esta pregunta se formuló con el fin de saber si los cuidadores eran capaces de reconocer una úlcera en primer estadio. La pregunta fue abierta y para su posterior codificación, se 
agruparon las repuestas según color, estado de la piel, olor y dolor, aunque estas dos últimas son minoritarias:

- Según color: se aprecia un elevado porcentaje del $52 \%$ en el que los cuidadores apreciaban como rojo el color de la úlcera en el primer estadío, un $26 \%$ apreciaban morada la piel, por último, las respuestas en las que la piel les parecía blanca o negra fueron de un $11 \%$ cada una.

19 SEGÚN COLOR.

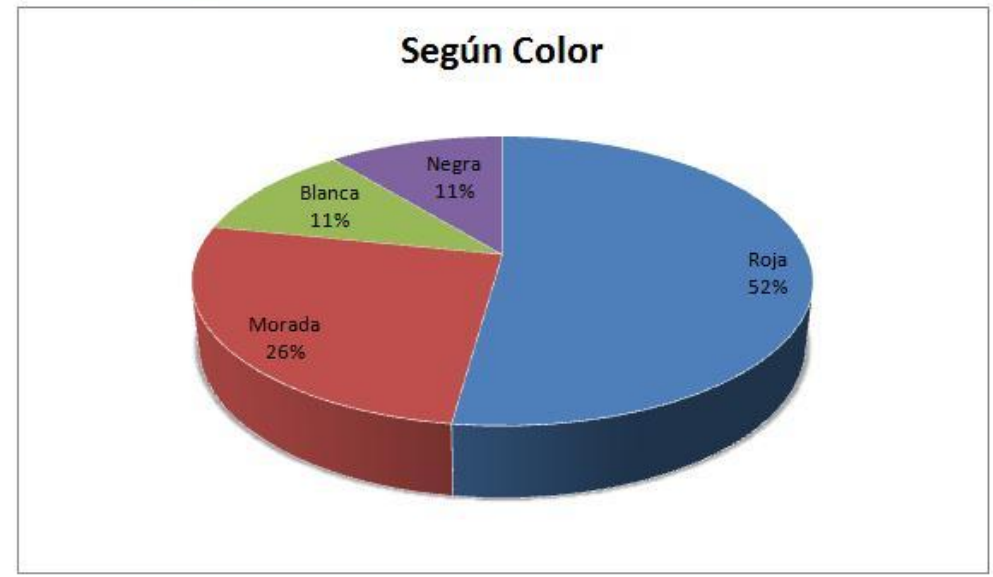

- Según el estado de la piel: el $36 \%$ de los cuidadores afirmaron que la piel se encontraba seca, el $27 \%$ llegaron a la conclusión de que la piel se encontraba delgada, es decir, con cierta pérdida de consistencia, un 14\% respondió que en la piel aparecían un serie de ampollas, al igual que otro $14 \%$ creían que la piel se encontraba irritada, un 5\% concibe la piel como edematosa, y por último un $4 \%$ la percibía como húmeda.

20 ESTADO DE LA PIEL.

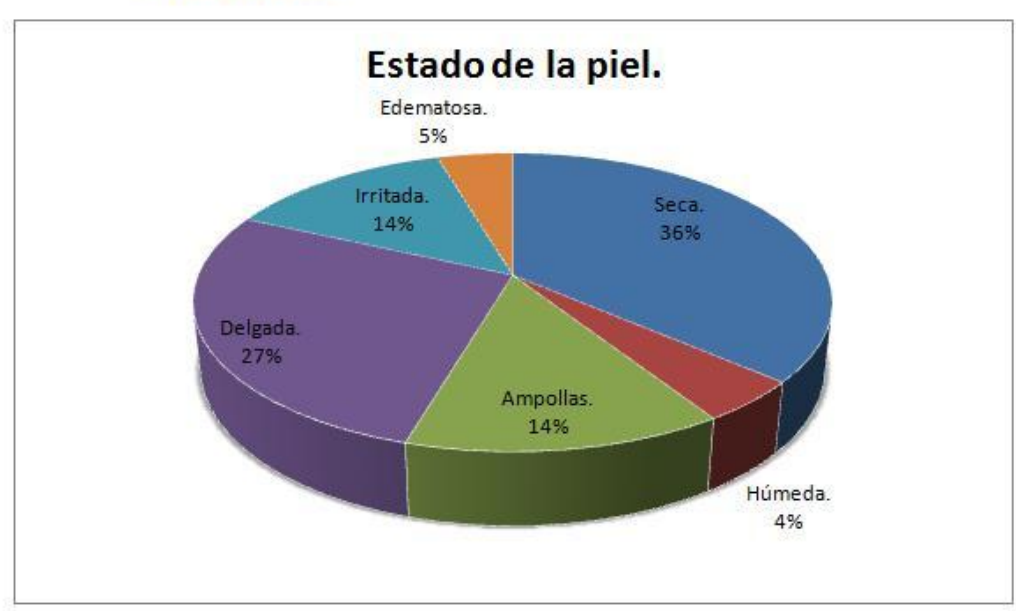

- Según el olor, sólo una persona contestó que "las úlceras se oscurecen y huelen mal".

- Según el dolor, unicamente dos cuidadores respondieron: " las úlceras son rojas, duelen y salen donde estan los huesos" y " son color rosaceo, que no palidecen y duelen". 


\section{DISCUSIÓN Y CONCLUSIONES}

El primer punto al que llegamos, tras observar la media de edad de los cuidadores, es que debemos de tener en cuenta que 53 años es una edad mediana, pero avanzada, por lo que en muchas ocasiones, las personas de esta edad también sufren ciertos problemas de salud. A esto añadiremos que la mayoría, el $86 \%$, son mujeres, con lo que también debemos sumar los posibles trastornos del climaterio. Asimismo, el hecho de que mayoritariamente sean una población activa y que en muchos casos trabajen también dentro del hogar.

Respecto al parentesco con el enfermo, se han producido cambios en los últimos 5 años relacionados con la economía de los familiares del enfermo, de esta manera, en la actualidad, ha habido un descenso de cuidadores formales, es decir, sin parentesco con el enfermo, por lo que ha habido un aumento de los cuidadores informales, ya que éstos no están remunerados. Se mantiene la tendencia del perfil del cuidador principal siendo mayoritariamente mujeres.

Nuestro primer objetivo, planteábamos estimar los conocimientos sobre los factores de riesgo de las úlceras por presión. Considerando las respuestas obtenidas en cuanto a los factores de riesgo podemos afirmar que los cuidadores son conscientes de que la inmovilidad es uno de los factores más importantes para la aparición de úlceras. De hecho, nos ha llamado la atención que el número de inmovilizados desciende conforme aumenta el tiempo de inmovilización, coincidiendo estos resultados con el estudio anteriormente citado (7). Existe cierta incertidumbre por parte de los cuidadores cuando se les aborda este tema, ya que la mayoría desconocen otros factores importantes como son: alimentación, humedad, higiene y adelgazamiento entre otros. Por esta razón, se debería trabajar más con los cuidadores sobre esta cuestión para aumentar los conocimientos sobre los factores de riesgo, mediantes la educación para la salud.

Dentro de los factores de riesgo, nos pareció interesante conocer si los cuidadores eran conscientes de las zonas comunes de aparición, ya que es donde hay que poner especial cuidado. Los cuidadores encuestados desconocen que las úlceras aparecen en cualquier prominencia ósea y sólo hacen hincapié en sacro y talones.

Al aumentar los conocimientos en la población sobre los factores de riesgo, automáticamente, estamos creando una defensa contra éstos, apareciendo de esta manera los métodos de prevención, que es nuestro segundo objetivo, evaluar los métodos de prevención que se utilizan para evitar úlceras por presión. Los cuidadores encuestados, llevan a cabo como métodos más eficaces de prevención: los cambios posturales e hidratación de la piel, restando importancia a otras medidas como son: masajes, nutrición y accesorios especiales. En cuanto a los cambios posturales la mayoría los realiza pero todavía existe un alto porcentaje, 39\%, que no los lleva a cabo; esto se debería de trabajar con educación para la salud junto con el tiempo entre cambios posturales ya que las cifras nos revelan que sólo un $38 \%$ del total los realiza correctamente.

En cuanto al uso de superficies de apoyo, los cuidadores están muy bien instruidos, ya que existe un alto porcentaje de cuidadores que aplican a sus enfermos medidas de protección especiales para acolchar las prominencias óseas.

Las cifras obtenidas con respecto al soporte nutricional hablan por sí solas, una gran cantidad de los encuestados desconoce que un cambio de alimentación pueda prevenir y/o favorecer la curación de upp. A este respecto, creemos que es tarea del equipo realizar 
actividades de educación sanitaria, instruyendo y adiestrando al cuidador en un soporte nutricional adecuado con respecto a las necesidades del enfermo.

Nuestro último objetivo es examinar la identificación de las úlceras por presión por parte del cuidador principal. La conclusión a la que hemos llegado ha sido que los cuidadores son capaces de reconocer las úlceras por: color, dolor, olor y estado de la piel. La mayoría de los encuestados coinciden en que debutan con tonos rojos o morados y el estado de la piel es seco y delgado, por ello utilizan como métodos de prevención la hidratación de la piel y el uso de superficies de apoyo.

Mediante actividades sanitarias instructivas al cuidador, junto con el cumplimiento de nuestros tres objetivos conseguiríamos que descendiese el número de veces que el cuidador acude con el enfermo al centro de salud.

Rescatando la hipótesis planteada de que existen unos conocimientos deficientes del cuidador principal en relación a las úlceras por presión, llegamos a la conclusión de que nuestra hipótesis se confirma.

\section{BIBLIOGRAFÍA REFERENCIADA}

1. INE. (pagina consultada el 4 de enero de 2010). Estimaciones de la población actual de España. [on-line]. Dirección

URL: http://www.ine.es/jaxiBD/tabla.do?per=01\&type=db\&divi=EPOB\&idtab=4

2. Abellán A. Indicadores demográficos. En: informe 2004. Las personas mayores en España. Observatorio de las personas mayores. Madrid: IMSERSO. CSIC; 2004. P. 4388.

3. Regueiro Martínez A.A, Ferreiro Cruz M.C, Gomara Villabona S.M, Perez- Vazquez A. Sobrecarga del cuidador principal del paciente en atención primaria. Atención Primaria. Julio- Agosto 2006; Volumen 38 (3):183-184.

4. Sanz Sanz M, Castillo Lizarraga M.J. El cuidador principal: manejo de los problemas de afrontamiento. Jano. Febrero-Marzo 2007. (Número 1639):43-44.

5. Ubiergo Ubiergo M.C, Regoyos Ruiz S, Vico Gavilán M.V, Reyes Molina R. El soporte de enfermería y la claudicación del cuidador informal. Revista de enfermería clínica. JulioAgosto 2005; 15(4).199-205.

6. Orem D. modelo de orem: conceptos de enfermería en la practica. Barcelona: massonsalvat; 1993

7. Vasco Uribe A. (pagina consultada el 4 de enero de 2010). Las ulceras por presión, la piel, el cuerpo y su entorno. Un problema del cuidado. [on-line]. Dirección URL: Http://www.aurasalud.com/articulos/art enfermeria/ulceras1.htm

8. Silvestre C, Domench L, Elizondo A, Erro J.I, Esparza C, Merino A, et al. (pagina consultada el 4 de enero de 2010). Evolución de la prevalencia de úlceras por presión en
el
Hospital
de
Navarra.
[on-line].
Dirección
URL:

http://www.cfnavarra.es/salud/anales/textos/vol22/n3/enfera.html

9. Blanco López J.L. Definición y clasificación de ulceras por presión. El peu. OctubreDiciembre 2003; 23(4).194-198.

10. Grupo Nacional para el Estudio y Asesoramiento en Ulceras Por Presión y Heridas Crónicas P. (GNEAUPP). Clasificación - estadiaje de úlceras por presión. Logroño, 2003.

11. Grupo Nacional para el Estudio y Asesoramiento en Ulceras Por Presión y Heridas Crónicas P. (GNEAUPP). Directrices generales sobre prevención de las ulceras por presión. Logroño, 2003.

12. NURE Investigación, (pagina consultada el 4 de enero de 2010) Protocolo de prevención y tratamiento ante úlceras por presión, [on-line]. Dirección URL: 
http://www.fuden.es/protocolos obj.cfm?ID PROTOCOLO=89\&paginacion=1

13. Juan $E$ Blümel $M$, Karina Tirado $G^{a}$, Claudia Schiele $M^{a}$, Gabriela Schönffeldt $F^{a}$, Salvador Sarrá C. Revista Médica de Chile. Validez de la escala de Braden para predecir úlceras por presión en población femenina. Mayo 004; 132(5): 595-600.

\section{BIBLIOGRAFÍA CONSULTADA}

- Delicado, M.V. Candel, E. Alfaro, A. López, M. García, C. Interacción de enfermería y cuidadoras informales de personas dependientes. Atención primaria. Marzo 2004; 33 (4): 193-199.

- Escalera, A. Mesonero, T. Caso clínico: cansancio en el rol de cuidadora. Metas de enfermería. Septiembre 2009; 12 (7): 57-62.

- Hospital de Bellvitge. Prevención y tratamiento de las úlceras por presión. Rol de enfermería. Marzo 2004; 27 (3): 53-55.

- Leyva-Moral, J.M. Mogueda-Marina, N. Necesidades psicosociales del cuidador informal. Rol de enfermería. Marzo 2008; 31 (3): 36-39.

- Moreno-Gaviño, L. Bernabeu-Wittel, M. Álvarez-Tello, M. Rincón, M. Bohórquez, P. Cassani, M. et al. Sobrecarga sentida por la figura del cuidador principal en una cohorte de pacientes pluripatológicos. Atención primaria. Abril 2008; 40 (4): 193-198.

- Nadal, M.J. Actualidad de la prevención de upp y ulceras vasculares. Rol de enfermería. Enero 2005; 28 (1): 50-54.

- Orta, M.A. Rodríguez, R.M. Prieto, J. Atención al cuidador principal desde atención primaria de salud. Metas de enfermería. Julio- agosto 2007; 10 (6): 18-22.

- Puig, M. Rodríguez, N, Valoración enfermera y calidad de vida en los cuidados domiciliarios. Metas de Enfermería. Julio- Agosto 2009; 12 (6): 63-67.

- Segovia, T. Pérez, M.E. Rol del cuidador principal en el cuidado del paciente anciano hospitalizado. Metas de enfermería. Septiembre 2001; 4 (38): 16-22.

- Sociedad Española de Geriatría y Gerontología. (13 de febrero de 2010). Perfil característico del cuidador, [on-line]. Dirección URL: http://www.segg.es/book/perfil/del/cuidador

- Zamora, J.J. Martínez, R. Puig, N. Lladó, M. Quílez, F. Programa de continuidad de cuidados al alta en una unidad de hospitalización a domicilio. Metas de enfermería. Abril 2009; 12 (3): 23-30.

- Zapata-Sampedro, M.A. Matute-Caballero. M. N. Gómez-Reina M.V. plan de cuidados enfermeros al cuidador informal. Caso clínico. Enfermería clínica. Mayo-junio 2007; 17 (3): 157-161. 


\section{ANEXOS}

ANEXO 1. ESCALA DE NORTON MODIFICADA

I ESCALA DE NORTON MODIFICADA

\begin{tabular}{|l|l|l|l|l|l}
\hline $\begin{array}{l}\text { Estado } \\
\text { Físico } \\
\text { General }\end{array}$ & $\begin{array}{l}\text { Estado } \\
\text { Mental }\end{array}$ & Actividad & Movilidad & Incontinencia & Puntos \\
\hline Bueno & Alerta & Ambulante & Total & Ninguna & 4 \\
\hline Mediano & Aasitico & Disminuida & $\begin{array}{l}\text { Camina con } \\
\text { ayuda }\end{array}$ & Ocasional & 3 \\
\hline Regular & Confuso & Muy limitada & Sentado & Urinaria o fecal & 2 \\
\hline Muy malo & $\begin{array}{l}\text { Estuporoso } \\
\text { comatoso }\end{array}$ & Inmóvil & Encamado & Urinaria y fecal & 1 \\
\hline
\end{tabular}

ANEXO 2. ESCALA EMINA

II ESCALA EMINA

\begin{tabular}{|c|c|c|c|c|c|}
\hline Nutrición & $\begin{array}{l}\text { Estado } \\
\text { Mental }\end{array}$ & Actividad & Movilidad & \begin{tabular}{|l|} 
Humedad \\
$\mathrm{R} / \mathrm{C}$ \\
Incontinencia
\end{tabular} & Puntos \\
\hline No ingesta & Comatoso & No deambula & Inmóvil & Urinaria y fecal & 3 \\
\hline Incompleta & $\begin{array}{l}\text { Letárgico } 0 \\
\text { hipercinético }\end{array}$ & $\begin{array}{l}\text { Siempre } \\
\text { precisa ayuda }\end{array}$ & $\begin{array}{l}\text { Limitación } \\
\text { importante }\end{array}$ & $\begin{array}{l}\text { Urinaria o fecal } \\
\text { habitual }\end{array}$ & 2 \\
\hline $\begin{array}{l}\text { Ocasionalmente } \\
\text { incompleta }\end{array}$ & $\begin{array}{l}\text { Desorientado } \\
\text { o apático o } \\
\text { pasivo }\end{array}$ & $\begin{array}{l}\text { Deambula con } \\
\text { ayuda }\end{array}$ & $\begin{array}{l}\text { Ligeramente } \\
\text { limitada }\end{array}$ & $\begin{array}{l}\text { Urinaria o fecal } \\
\text { ocasional }\end{array}$ & 1 \\
\hline Correcta & Orientado & Deambula & Completa & No & 0 \\
\hline
\end{tabular}


ANEXO 3. ESCALA DE BRADEN

\section{ESCALA DE BRADEN}

Figura 1. Escala de Braden

\begin{tabular}{|c|c|c|c|c|}
\hline $\begin{array}{l}\text { Pencepción Sensonial } \\
\text { Capacidad de re spuesta } \\
\text { a extimulos dolorosos }\end{array}$ & $\begin{array}{l}\text { 1. Limitado } \\
\text { completumente }\end{array}$ & $\begin{array}{l}\text { 2. Muy } \\
\text { limitado }\end{array}$ & $\begin{array}{l}\text { 3. Limitado } \\
\text { levemenie }\end{array}$ & $\begin{array}{l}\text { 4. Sin } \\
\text { impedimeno }\end{array}$ \\
\hline $\begin{array}{l}\text { Humedad } \\
\text { Grado de humedad de piel }\end{array}$ & $\begin{array}{l}\text { 1. Constantemente } \\
\text { húmeda }\end{array}$ & $\begin{array}{l}\text { 2. Muy } \\
\text { húmeda }\end{array}$ & $\begin{array}{l}\text { 3. Ocasionalmente } \\
\text { húmeda }\end{array}$ & $\begin{array}{l}\text { 4. Raramente } \\
\text { húmeda }\end{array}$ \\
\hline $\begin{array}{l}\text { Actividad } \\
\text { Grado de actividad fisica }\end{array}$ & $\begin{array}{l}\text { 1. Confinado } \\
\text { a la cama }\end{array}$ & $\begin{array}{l}\text { 2. Confinado } \\
\text { a la slla }\end{array}$ & $\begin{array}{l}\text { 3. Ocasionalmente } \\
\text { camina }\end{array}$ & $\begin{array}{l}\text { 4. Camina } \\
\text { frecusrtemente }\end{array}$ \\
\hline $\begin{array}{l}\text { Movifidad } \\
\text { Contel de posicion corporal }\end{array}$ & $\begin{array}{l}\text { 1. Completumenve } \\
\text { inmbvil }\end{array}$ & $\begin{array}{l}\text { 2. Muy } \\
\text { limitada }\end{array}$ & $\begin{array}{l}\text { 3. Levemente } \\
\text { limitada }\end{array}$ & $\begin{array}{l}\text { 4. Sin } \\
\text { limitaciones }\end{array}$ \\
\hline $\begin{array}{l}\text { Nutricón } \\
\text { Patrón de ingesa alimentaria }\end{array}$ & $\begin{array}{l}\text { 1. Completamenve } \\
\text { inadecuada }\end{array}$ & $\begin{array}{l}\text { 2. Probablemente } \\
\text { inadecuada }\end{array}$ & 3. Adecuada & 4. Excelenve \\
\hline $\begin{array}{l}\text { Fricción y roce } \\
\text { Roce de piel con sibanas }\end{array}$ & 1. Presente & $\begin{array}{l}\text { 2. Povencialmente } \\
\text { presenve }\end{array}$ & 3. Ausente & \\
\hline
\end{tabular}

Se considera como nesgo de desarrollar úloeras un puntaje menor o igual a 16 .

\section{ANEXO 4.}

\section{CUESTIONARIO}

1. ¿Qué edad tiene?

2. ¿Sexo?

3. ¿Qué relación tienes con el enfermo?

4. ¿Qué nivel de estudios tiene?
a. Primaria.
b. Secundaria.
c. Bachillerato.
d. Universitarios.

5. ¿Ha sufrido el anciano/a en los últimos meses úlceras?
a. Si.
b. No. 
6. ¿Cuáles son los motivos físicos que provocan la aparición?
a. Presión.
b. Fricción.
c. Cizallamiento.
d. Humedad.

7. ¿Cuáles son los factores de riesgo que afectan en la aparición de upp?
a. Edad avanzada.
b. Patologías asociadas.
c. Inmovilidad.
d. Fármacos.
e. Situación sociofamiliar.
f. Higiene.
g. Arreglo cama.
h. Exceso humedad.

8. ¿Crees que existe relación entre las patologías de un anciano y las upp?
a. Si.
b. No.

9. ¿Conoces las zonas en las que hay que poner especial cuidado para evitar la aparición de upp?
a. Si.
b. No.

10. ¿Utilizas algunos métodos de prevención frente a upp?
a. Cambios posturales.
b. AGHO.
c. Superficie de apoyo.
d. Higiene.
e. $\ldots$

11. ¿Crees que la higiene y el arreglo de la cama se considera un método de prevención o por el contrario no influye?
a. Si influye.
b. No influye.

12. ¿Fomentar la movilidad y actividad del paciente disminuye la probabilidad de padecer upp?
a. Si.
b. No. 
13. ¿Realiza cambios posturales?
a. Si.
b. No.

14. ¿Con que frecuencia?
a. Cada 2 horas.
b. Cada 4 horas.
c. Cada 6 horas.
d. Cada 12 horas.
e. No realiza.

15. ¿Utiliza alguna superficie de apoyo especial?
a. Si.
b. No.

16. ¿De qué tipo?
a. Cojines.
b. Colchón antiescaras.
c. Colchón látex.
d. Viscoelasticos.
e. Colchonetas.

17. ¿Presenta más importancia el uso de superficies de apoyo o la realización de cambios posturales?
a. Superficie de apoyo.
b. Cambios posturales.
c. Ambas.

18. ¿Utiliza soporte nutricional especial durante la curación de la upp?
a. Si.
b. No.

19. ¿Cuál?
a. Aumento de calorías.
b. Aumento de proteínas.
c. Aporte hídrico.
d. Aumento de minerales, vitaminas y amino ácidos.

20. ¿Qué características presenta la piel en la aparición de upp? 
ISSN 1695-6141

๑ COPYRIGHT Servicio de Publicaciones - Universidad de Murcia 